\title{
ADDITIVE MANUFACTURING PROCESS PARAMETER INFLUENCE ON MECHANICAL STRENGTH OF ADHESIVE JOINTS, PRELIMINARY ACTIVITIES
}

\author{
Mattia Frascio ${ }^{a, *}$, Lorenzo Bergonzi ${ }^{b}$, Michal Jilich $^{a}$, \\ Fabrizio Moroni ${ }^{b}$, Massimiliano Avalle $^{a}$, Alessandro Pirondi ${ }^{b}$, \\ Margherita Monti $^{a}$, MAtteo Vettori ${ }^{c}$ \\ ${ }^{a}$ Universitá degli studi di Genova, Via Opera Pia 15a, 16145 Genova, Italy \\ ${ }^{b}$ Universitá degli studi di Parma, Via Universitá 12, 43121 Parma, Italy \\ c MaCh3D srl, Strada S. Nicoló 19, 43126 Parma, Italy \\ * corresponding author: mattia.frascio@edu.unige.it
}

\begin{abstract}
The work illustrates how building parameters of the Additive Manufacturing process Fused Filament Fabrication can affect not only the mechanical properties but also the surface wettability and morphology. Wettability and morphology are relevant factors in bonded joints performance. Advantages of polymeric additive manufacturing are to allow a re-design of components with locally controlled properties and integrated functions. Major limitations are related to the lack of material testing standardization and constraints due to the build volume and to the object orientation for printability: the latter problems can be addressed with the use of bonded joints that allow to create bigger assemblies from smaller parts optimally designed to take advantage of material orthotropy and without the structural drawbacks. In this study, two materials are considered, acrylonitrile butadiene styrene and polylactideacid. Wettability, surface morphology and mechanical strength have been determined at different combinations of nozzle temperature, print speed and layer thickness.
\end{abstract}

KEYWORDS: Additive manufacturing, fused filament fabrication, bonded joints, surface characterization.

\section{INTRODUCTION}

In this work it is presented the identification by experimental testing of the influence of the 3D printing process parameters on components characteristics, as tensile strength and surface finishing, relevant for the bonded joint process. It is implemented using the design of experiments DOE to be able to investigate at the same time the effects of three factors (temperature of nozzle $\mathrm{T}$, velocity of deposition $\mathrm{V}$, thickness of the layer $\mathrm{H}$ ) at three levels. The monitored output are the mechanical properties, the wettability and the morphology of 3D printed specimens. This allowed to obtain a more comprehensive reference to design a bonded joint that use the fused filament fabrication (FFF) for the build of the adherends. The selected materials for the adherends are commercial polymers supplied for additive manufacturing purpose in order to produce data using polymer with chemical composition, molecular weight and dispersity developed for this technology. The material selection took in account many factors as the rate of utilization of the material in FFF application, the mechanical properties, the durability and the processability. Therefore acrylonitrile butadiene styrene (ABS) and polylactideacid (PLA) were chosen. The experimental data are analysed using statistical methods, the analysis of variance (ANOVA), to achieve a better understating of the dependence on printing parameters of the mechanical properties, wettability and morphology measured.

\section{Motivation}

The advantages of Additive Manufacturing (AM) to allow a re-design of components with locally controlled properties [1] and integrated functions [2] are yet insufficient to balance the technological drawbacks such as the lack of material standardization and constraints due to the build volume and to the object orientation for printability. The latter problems can be addressed with the use of bonded joints. Bonded joints allow to create bigger assemblies from smaller parts optimally designed to take advantage of material orthotropy without structural drawbacks. While it is a wellknown fact that the process parameters of the AM technology FFF are relevant for the mechanical properties [3, 4] the influence of the build set up on the surface characteristics, as wettability and morphology, has not been investigated yet. However that are relevant factors in bonded joints performance [5].

When components made by $3 \mathrm{D}$ printing are assembled by bonding, the method of bonding and in particular the parameters of the bonding, are chosen on the base of the mechanical properties, wettability and morphology of the components to be assembled. It is common to refer to the specifications provided by the supplier of the filaments but it is proven [2] that these change significantly with the parameters of the printing process. So can be concluded that a 

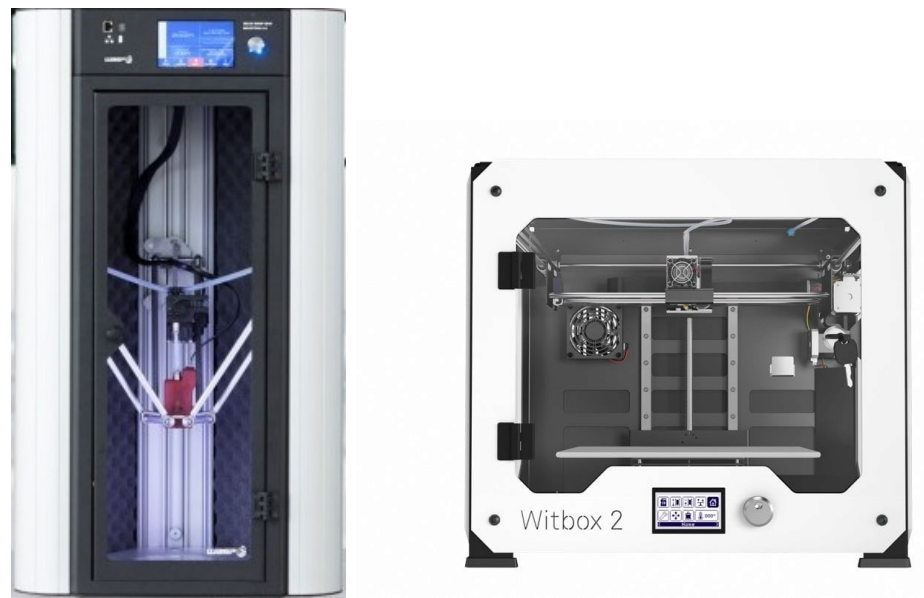

Figure 1. The used 3D printers, the delta WASP 4070 Industrial by Wasp and the portal Witbox by bq
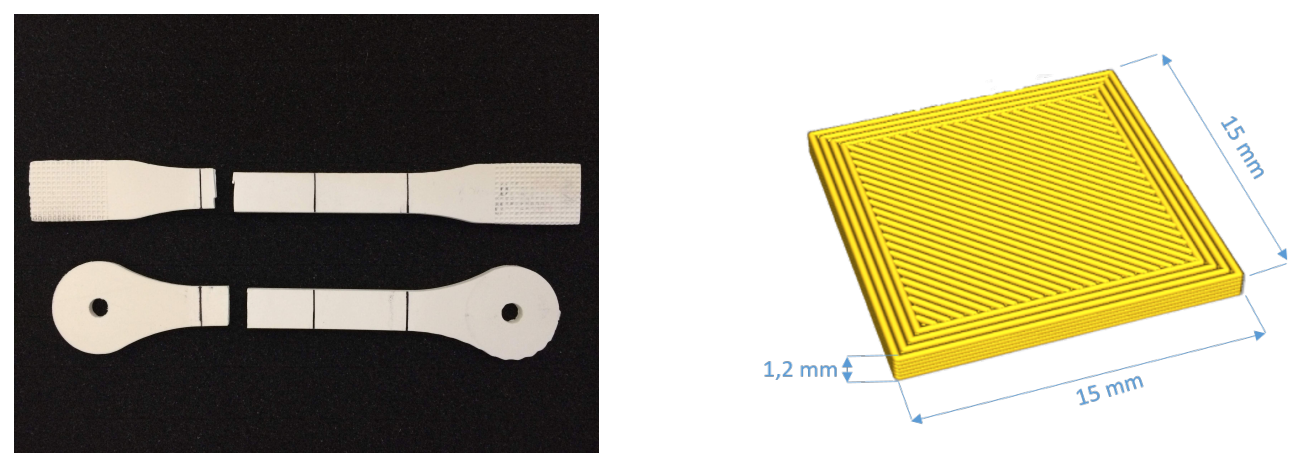

Figure 2. The MaCh3D specimen specimen compared to the BS EN ISO 527-2:2012 specimen and the prismatic specimen used for wettability and morphology characterizations

bonding method for highest mechanical performance of the joint must be defined on actual properties to be measured on printed samples [6, 17.

\section{MATERIALS}

\subsection{D PRINTERS AND PROCESS PARAMETERS}

Two fused filament fabrication FFF 3D printing machines are used, a Witbox1FDM by bq and a WASP 4070 industrial by Wasp (figure 1).

The fused FFF process consists in a liquefied filament of polymer pushed through a heated nozzle. The geometry is build by stacking layers of opportunely deposition patterns of the nozzle. The nozzle can be moved by different kinematic schemes of actuation: by portal scheme (ex. the one adopted by bq for the Witbox1FDM) nominally higher geometrical accuracy is achievable, by delta (ex. technical solution adopted by the Wasp) is possible to use higher print speed to cut the build time instead [8]. The choice to use both the $3 \mathrm{D}$ printers is done to test the kinematic schemes in order to verify if there is any actual influence on the measured properties. Specimens of two materials are built using both $3 \mathrm{D}$ printers. Acrylonitrile butadiene styrene (ABS) is selected in relation to the compatibility characterization with different adhesives that is being performed [9, 10]; moreover it can be used when durability is a requirement for the application. Polylacticacide (PLA) is chosen as it is the most used in FFF, but mostly because it is a biodegradable material [11 13].

The solid models are designed using Creo Parametric 2.0 software, different geometries of specimens are used for the different characterizations: the MaCh3D tensile test specimen for tensile test and prismatic specimens for morphology and wettability characterization (figure 2). The slicing and building parameters set up is done using the Ultimaker Cura software. The placement of the specimens is with the dominant length along the machine $\mathrm{x}$-axis, resulting in a $\pm 45^{\circ}$ raster orientation, configuration compliant to the bonding application [1. Then multiples Gcode are generated at the same time to obtain controlled variations of the investigated parameters, nozzle temperature, deposition speed and layer height, preventing the influence of undesired variations in the set-up. These parameters are chosen as relevant factors for the experiments because expected to affect the polymer crystallinity, the coalescence and the intra-layers 


\begin{tabular}{lcccccc}
\hline Material & \multicolumn{3}{c}{ ABS } & \multicolumn{3}{c}{ PLA } \\
\hline Factor level & 1 & 2 & 3 & 1 & 2 & 3 \\
Nozzle temperature T $\left({ }^{\circ} \mathrm{C}\right)$ & 230 & 240 & 250 & 200 & 215 & 230 \\
Deposition speed $(\mathrm{mm} / \mathrm{s})$ & 30 & 75 & 120 & 30 & 75 & 120 \\
Layer height H $(\mathrm{mm})$ & 0.1 & 0.2 & 0.3 & 0.1 & 0.2 & 0.3 \\
\hline
\end{tabular}

TABLE 1. Factors and levels

adhesion [13. Three levels for each relevant factor are investigated, values are set on the basis of previous experiences 4, 14, and considering the ranges indicated in the manufacturers data sheets (table 1). Worth nothing that the building time is highly affected by the specimen volume, the deposition speed and the layer height. By evaluating the needed accuracy of data analysis and by the building time esteem of each set-up, the design of experiments DOE is done. It led to a Taguchi Orthogonal Array experiment for tensile testing and to a full factorial design for wettability and morfology characterization.

\subsection{Characterization Set-up}

The mechanical testing is performed using MaCh3D, a miniaturized universal testing machine (figure 3) developed for products and materials certification directly on the production site [14, peculiarity relevant for additive manufacturing. Indeed additive manufacturing needs the development of cost effective and transportable tools. Tensile test experiments are performed on 9 sets of three specimens (figure 2 ) built varying the printing set-up as described in the design of experiments section.

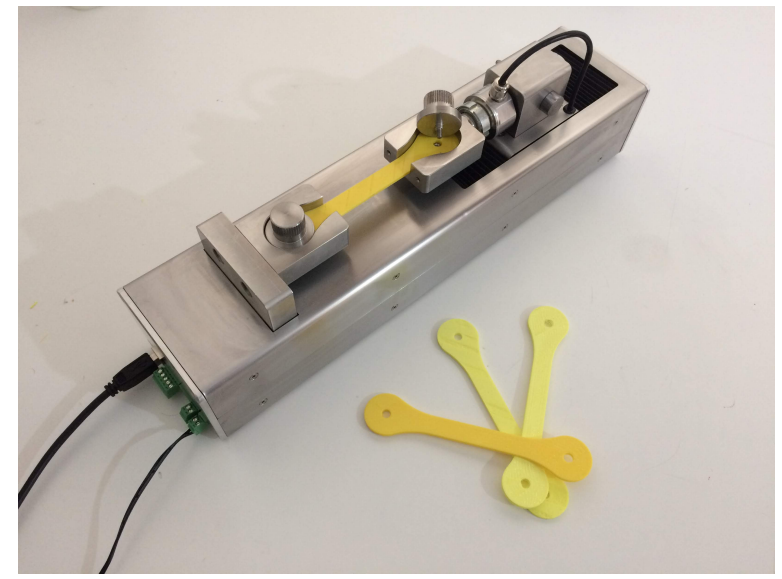

Figure 3. The MaCh3D miniaturized testing machine and $\mathrm{MaCh} 3 \mathrm{D}$ specimen

The wettability characterization is performed on the top layer of the prismatic specimens (figure 22 using a Milli-Q sessile drop tester with deionized water. Static contact angle measurements, measured where the liquid-vapor interface meets the solid interface, are performed with a custom made set-up based on the Dino-lite microscope (figure 4). The choice to measure the static contact angle is justified by the bonding application as the static contact angle reflects the relative strength of the molecular interaction at the interfaces. Specimens surfaces were cleaned using ethanol alcohol diluted in $30 \%(\mathrm{w} / \mathrm{w})$ deionized water solution. Images of the drop were taken by microscope from two different positions to consider the asymmetrical shape caused by the orthotropic surface morphology of the layer (figure 5).

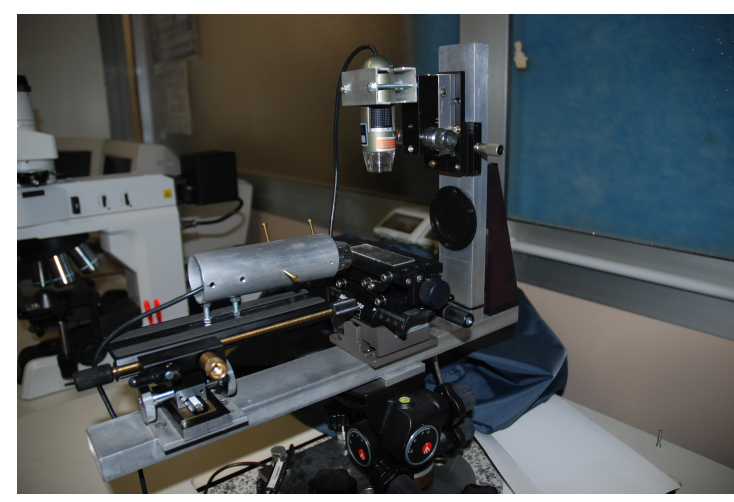

FIGURE 4 . The wettability measurement set-up

The roughness parameters were measured on the same prismatic specimens (figure 2) used to characterize the wettability as it is a well known fact that wettability is influenced also by the morphology [15. Specimens morphology were investigated by scanning the surface of top layer according to the built direction because it is the one affected by the printing parameters; the bottom surface have a finishing related mostly to the build plate. Measurements are performed compliant to EN ISO 25178 - 600:2019 using a CCI Taylor-Hobson 3D optical profilometer. The areal roughness parameter arithmetic mean height roughness is used in order to characterize surface morphology. The arithmetic mean height roughness, Sa, quantifies the deviations in height of the surface points in relation to the mean reference plane.

\section{Experimental Methods}

\subsection{Design of eXPeriments DOE}

Design of experiment (DOE) is a systematic approach to process optimization [16. In this work it is used to 

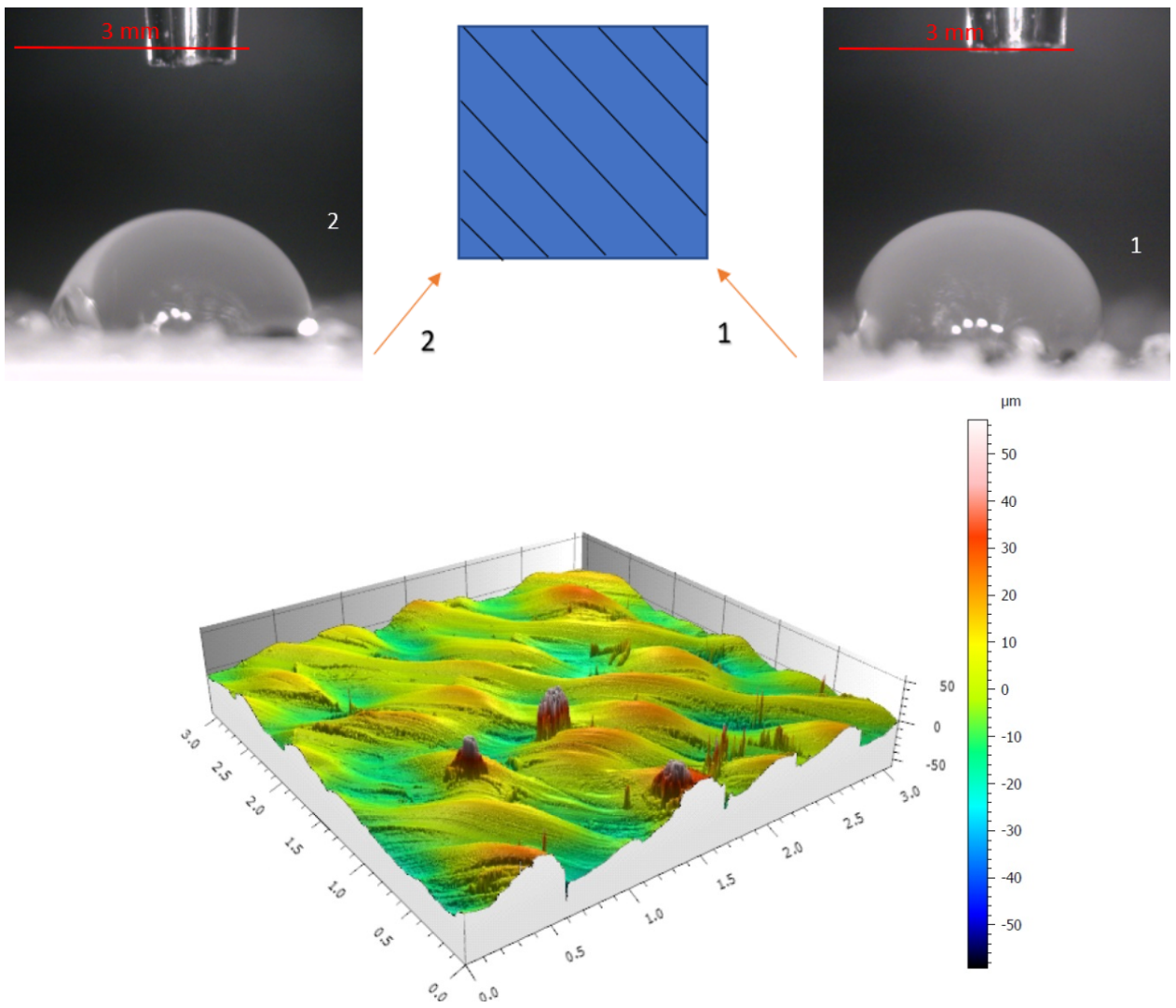

FiguRE 5. Wettability and morfology measurements

determine how the factors (nozzle temperature, deposition speed and layer height), affect the response variables (the mechanical and surface properties). Each factor is experimented on three discrete levels as described in Table 1. Full factorial design consists in testing all the possible combinations of all levels of the factors varying one factor at a time. It is feasible for morphology and wettability characterization due to the use of miniaturized specimens despite the high number of experiments required [17]. It is chosen considering the high precision of the estimation of the effects of the factors and interactions between factors on the response variables. The Taguchi method is selected to study the effect of the print parameters on tensile testing output; it also introduces the use of orthogonal array (OA) to reduce the number of experiments despite the same number of factors and levels investigated. The orthogonal array (OA) is selected on the degrees of freedom of the problem. The degrees of freedom are the minimum number of independent experiments to be conducted [18; as the degrees of freedom are one for the mean value and two each for the remaining factors the Taguchi based $\mathrm{L}_{9} \mathrm{OA}$ is chosen and nine experiment configurations for each material are carried out (table 2).

Repetitions and confounding, randomized testing sequence, are applied for the specimens testing in order to reduce experimental errors. The tensile test data are processed with the larger is best (equation 1) signal-to-noise-ratio (SNR) to obtain from the several

\begin{tabular}{cccc}
\hline Experiment & Factor 1 & Factor 2 & Factor 3 \\
\hline 1 & 1 & 1 & 1 \\
2 & 1 & 2 & 2 \\
3 & 1 & 3 & 3 \\
4 & 2 & 1 & 3 \\
5 & 2 & 2 & 1 \\
6 & 2 & 3 & 2 \\
7 & 3 & 1 & 2 \\
8 & 3 & 2 & 3 \\
9 & 3 & 3 & 1 \\
\hline
\end{tabular}

TABLE 2. Taguchi orthogonal array $\mathrm{L}_{9}$

repetitions the measure of the variation present due to the controlled factors.

$$
\mathrm{SNR}=-10 \log \left(\frac{\sum \frac{1}{y_{i}^{2}}}{n}\right)
$$

Where $y_{i}$ is the observed response value for the i-th trial and $\mathrm{n}$ the number of observations for the trial. The advantage of the use of the balanced orthogonal array (OA) is that the data analysis is simplified, ex. the effects can be estimated independently. The main effect, the change in response produced by the change of the factors levels can be computed using

$$
E_{t}=F_{h}-F_{l}
$$


where $E_{t}$ is the main effect, $F_{h}$ is the average response at high level, $F_{l}$ is the average response at low level. The interaction effect, the change in response due to the combined effect of more than one factor, is computed as half of the difference between the effect of one factor $F_{1}$ at high level of the other factor $F_{2}$ and the effect of the same factor at low level of the other factor. The interaction effect is written in symbol as $F_{1} \times F_{2}$.

\subsection{Analysis of Variance ANOVA}

The analysis of variance (ANOVA) is performed to determine which of these effects are statistically relevant [16-18. The analysis of variance (ANOVA) tool allow to sub-divide the variation in the data in components that can be addressed to the main effects, the interaction effects and errors. In particular the F-ratio test allow to determine if a factor is significant. The effect of each factor or interaction relative to the error is significant if the $\mathrm{F}$ factor, the ratio between the effect variance and the error variance, is higher than a critical $F$. The value of $F$ critical is given by the degrees of freedom of the experiment. The information from DOE and ANOVA are used to determine the process parameters set up for specimens with enhanced tensile and surface characteristics.

\section{Results AND Discussion}

Experimental data are reported and elaborated to extrapolate main and interaction effects, F-statistic values for ANOVA and Signal to Noise Ratio (SNR). These are relevant information to understand the effects of factors on the monitored output in order to define the build configurations to take advantage of these effects. The results are shown in the pictures, in which the main effects are abbreviated as $\mathrm{T}$ for the nozzle temperature, $\mathrm{V}$ for the deposition speed and $\mathrm{H}$ for the layer height. In the $\mathrm{x}$ axis is reported the level.

\subsection{Tensile testing Results}

Tensile testing are performed with three repetitions for each level. Experimental ultimate tensile strength ranges from a minimum of $20 \mathrm{MPa}$ to a maximum of $40 \mathrm{MPa}$. The main effect analysis and the interaction effect for tensile data are reported in figure 6. For the ABS there is an evident dependence with the nozzle temperature and a correlation between velocity and both temperature and layer height. For PLA there are significant effects of the temperature and velocity. Relevant interaction between layer height and both temperature and velocity is noticed.

ANOVA analysis is performed. The $\mathrm{F}$ critical value for $95 \%$ confidence interval is 2.51. The values in table 3 compared to the $\mathrm{F}$ critical, confirm the information drawn from the main effect analysis.

The signal-to-noise-ratio SNR analysis (figure 7) is performed (table 6) to identify a best building set up based on relevance of main effect and building time

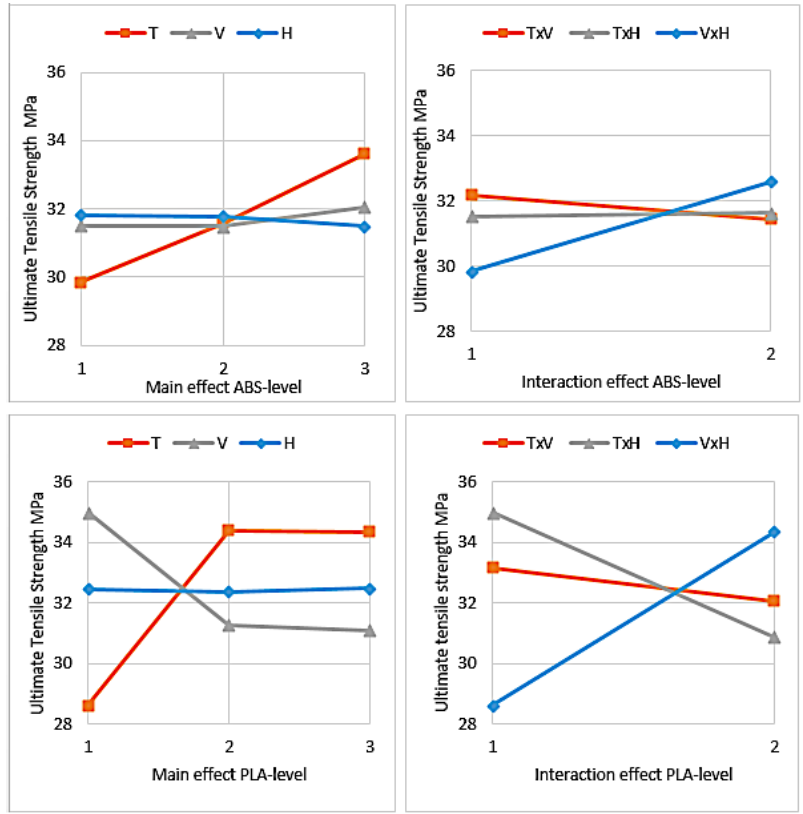

Figure 6. Main and interaction effect on ultimate tensile strength

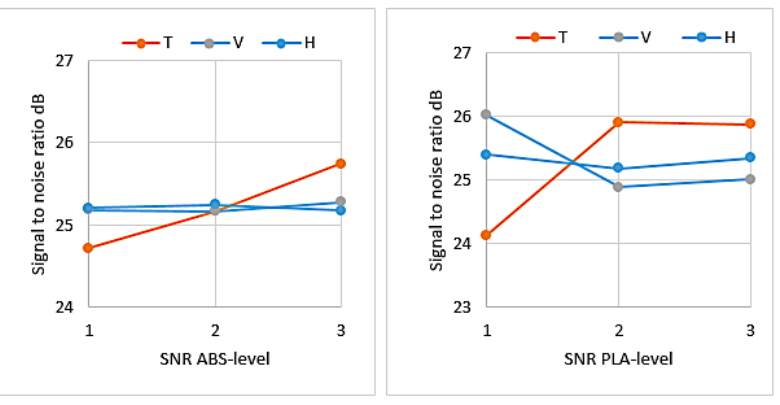

FigURE 7. signal-to-noise-ratio analysis on ultimate tensile strength

\begin{tabular}{lcc}
\hline Source & F - ABS & F - PLA \\
\hline Nozzle temperature & 6.78 & 27.68 \\
Deposition speed & 0.19 & 12.26 \\
Layer heigh mm & 0.06 & 0.01 \\
\hline
\end{tabular}

TABLE 3. UTS F factors.

minimization, a critical factor for additive manufacturing [8].

\subsection{Wettability Results}

Wettability testing is performed, nine repetitions for each specimen, experimental static contact angle ranges from a minimum of $50^{\circ}$ to a maximum of $130^{\circ}$. The values of the main effect and of the interaction effect are reported in figure 8 . For both ABS and PLA, the deposition speed has the most relevant effect.

The ANOVA analysis is performed and the computed $\mathrm{F}$ values are shown in table 4 . The $\mathrm{F}$ critical for $95 \%$ confidence interval is 1.84 . The comparison of the values to $\mathrm{F}$ critical confirm the outcome of the 


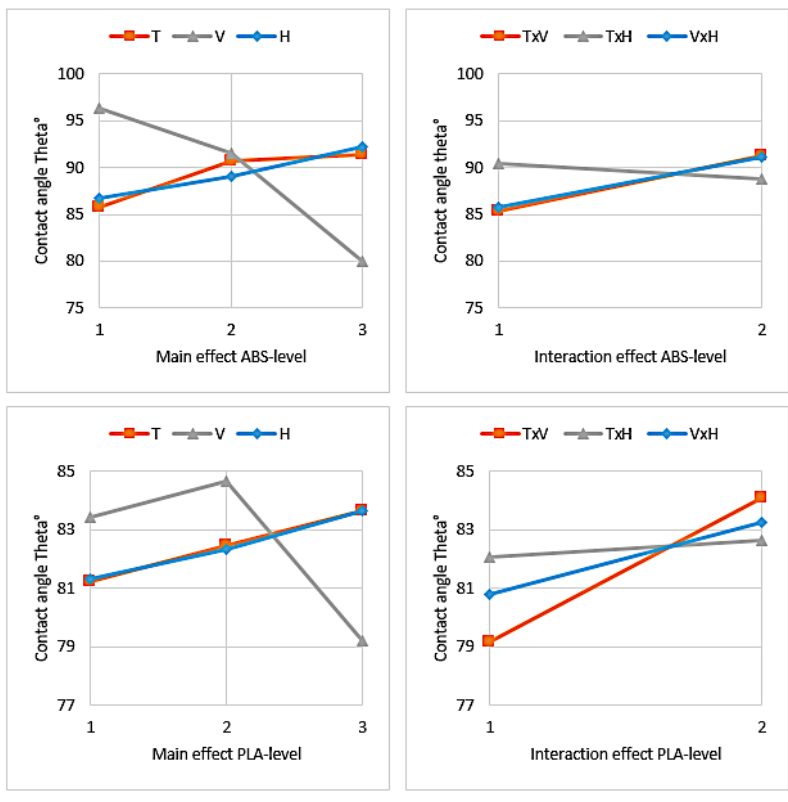

FiguRE 8. Main and interaction effect on wettability

main effect analysis.

\begin{tabular}{lcc}
\hline Source & F - ABS & F - PLA \\
\hline Nozzle temperature & 0.74 & 0.21 \\
Deposition speed & 5.24 & 1.3 \\
Layer heigh & 0.57 & 0.23 \\
\hline
\end{tabular}

TABLE 4. Wettability F factors

\subsection{Morphology Results}

Morphology testing is performed on three specimens and three repetitions for each 3D printing set-up. Experimental Sa ranges from a minimum of $7 \mu \mathrm{m}$ to a maximum of $26 \mu \mathrm{m}$. The main effect analysis and interaction effect analysis are reported in figure 9 For ABS the height $\mathrm{H}$ has a relevant effect, and, for PLA, both $\mathrm{T}$ and $\mathrm{V}$ are relevant.

The ANOVA analysis is performed, results are reported in table 5. The value of the $\mathrm{F}$ critical for the $95 \%$ confidence interval is 1.84 . The computed values in table 5 compared to $\mathrm{F}$ critical confirm the information drawn from the main effect analysis.

\begin{tabular}{lcc}
\hline Source & F - ABS & F - PLA \\
\hline Nozzle temperature & 0.38 & 2.05 \\
Deposition speed & 1.15 & 17.15 \\
Layer heigh & 2.6 & 1.05 \\
\hline
\end{tabular}

TABlE 5. Morphology F factors

\section{Conclusions}

Building parameters for maximum ultimate tensile strength and maximum and minimum values of wettability and roughness are identified (table6). Using the

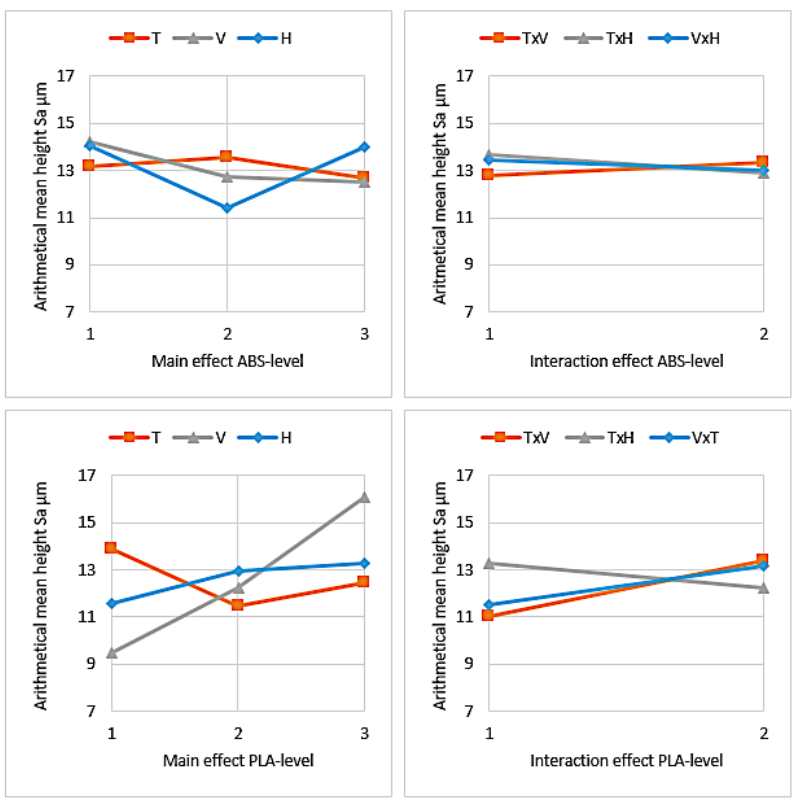

Figure 9. Main and interaction effect on morphology

F-test the relevant effects have been identified. The different kinematic schemes do not affect the measured output. The showed set-up in the table 6 is drafted according to the relevant effects and in order to minimize the build time, another critical issue for additive manufacturing. Worth nothing to notice that ABS has the maximum ultimate tensile strength UTS for the shorter build time set-up and that the two materials require sundry set-up to obtain the desired enhanced surface characteristics. Other works investigated the adherends materials compatibility with many adhesives or experiencing adherends modification through additive manufacturing as surface tailoring with patterns or infill variations. The results presented in this work are the preliminary activities needed to investigate further the actual influence of the additive manufacturing (AM) process parameters on mechanical strength of adhesive joints. 


\begin{tabular}{lcccccccccc}
\hline & \multicolumn{1}{c}{ Factor level } \\
\cline { 2 - 11 } Output & ABS- & \multicolumn{1}{c}{ ABS-THETA } & \multicolumn{2}{c}{ ABS-Sa } & \multicolumn{2}{c}{ PLA- } & \multicolumn{2}{c}{ PLA-THETA } & \multicolumn{2}{c}{ PLA-Sa } \\
& UTS & $\min$ & $\max$ & $\min$ & $\max$ & UTS & $\min$ & $\max$ & $\min$ & $\max$ \\
\hline Nozzle temperature (celsius) & 3 & 1 & 2 & 2 & 1 & 2 & 3 & 1 & 1 & 3 \\
Deposition speed (mm/s) & 3 & 1 & 3 & 2 & 3 & 1 & 1 & 2 & 2 & 1 \\
Layer heigh (mm) & 3 & 1 & 3 & 3 & 3 & 3 & 2 & 1 & 1 & 2 \\
\hline
\end{tabular}

TABLE 6. Configurations for single lap experimental investigation

\section{ACKNOWLEDGEMENTS}

This research was supported by Universitá degli studi di Genova, Universitá degli studi di Parma and MaCh3D srl.

\section{REFERENCES}

[1] L. Li. Composite modeling and analysis for fabrication of fdm prototypes with locally controlled properties. Journal of Manufacturing Processes 4:S129-S141, 2002. DOI:10.1016/S1526-6125(02)70139-4

[2] M. Jilich. Development of a gripper for garment handling designed for additive manufacturing. Proc IMechE Part C 1-12, 2019. DOI: $10.1177 / 0954406219857763$

[3] D. Popescu. Fdm process parameters influence over the mechanical properties of polymer specimens : A review. Polymer Test 69:157-166, 2018. DOI:10.1016/j.polymertesting.2018.05.020

[4] M.Frascio. Fatigue strength of plastics components made in additive manufacturing: first experimental results. 12, pp. 32-43. 2018. DOI:10.1016/j.prostr.2018.11.109

[5] D. Dillard. The mechanics of adhesion, vol. 2. 2002.

[6] B. M. Tymrak. Mechanical properties of components fabricated with open-source 3-d printers under realistic environmental conditions. Materials \& Design 58:242-246, 2014. DOI:10.1016/j.matdes.2014.02.038

[7] J. Kotlinski. Mechanical properties of commercial rapid prototyping materials. Rapid Prototyping Journal 20:499-510, 2014. DOI:10.1108/RPJ-06-2012-0052.

[8] I. Gibson. Additive Manufacturing Technologies, 3D Printing, Rapid Prototyping, and Direct Digital Manufacturing. 2015. DOI:10.1007/978-1-4939-2113-3.

[9] J.M.Arenas. Multi-criteria selection of structural adhesives to bond abs parts obtained by rapid prototyping. Int $J$ Adhes Adhes 33:67-74, 2012. DOI:10.1016/j.ijadhadh.2011.11.005.

[10] D.Espalin. Analysis of bonding methods for fdm-manufactured parts. 21st Annu Int Solid Free Fabr Symp-An Adit Manuf Conf 21, 2010.

[11] G. Wypych. Handbook of Polymers. 2016.

[12] C. Moliner. Mechanical and thermal performance of pla and phbv- based biopolymers as potential alternatives to pet. Chemical Engineering Transactions 57:1417-1422, 2017. DOI:10.3303/CET1757237.

[13] V. Srinivas. Correlating molecular and crystallization dynamics to macroscopic fusion and thermodynamic stability in fused deposi-tion modeling; a model study on polylactides. Polymer 142:348-355, 2018.

DOI:10.1016/j.polymer.2018.03.063
[14] L. Bergonzi. Numerical and experimental validation of a non-standard specimen for uniaxial tensile test.

Procedia Struct Integr 12:392-403, 2018. DOI:10.1016/j.prostr.2018.11.078

[15] K. Kubiak. Wettability versus roughness of engineering surfaces. Wear 271:523-528, 2011. DOI:10.1016/j.wear.2010.03.029

[16] J.Antony. Experimental quality, a strategic approach to achieve and improve quality. 2000. DOI:10.1007/978-1-4615-5293-2.

[17] V. Srinivas. Application of taguchi method for optimization of process parameters in improving the surface roughness of lathe facing operation.

International Refereed Journal of Engineering and Science 1:13-19, 2012.

[18] B.M.Gopalsamy. Taguchi method and anova, an approach for process parameters optimization of hard machining while machining hardened steel. Journal of Scientific and industrial Research 68:686-695, 2009. 